\section{$\zeta$}

\title{
Pulmonary microvascular architecture in hereditary haemorrhagic telangiectasia
}

\author{
Maximilian Ackermann, ${ }^{1}$ Steven J Mentzer, ${ }^{2}$ Wilfried Roth, ${ }^{3}$ Urban Geisthoff, ${ }^{4}$ \\ Erich Stoelben ${ }^{5}$
}

1 Institute of Functional and Clinical Anatomy, University Medical Center of the Johannes Gutenberg University Mainz, Mainz, Germany

2Division of Thoracic Surgery, Department of Surgery, Brigham and Women's Hospital, Harvard Medical School, Boston,

Massachusetts, USA

${ }^{3}$ Institute of Pathology, University Medical Center of the Johannes Gutenberg University Mainz, Mainz, Germany

${ }^{4}$ Department of

Otorhinolaryngology, Essen

University Hospital, Essen,

Germany

DDepartment of Thoracic Surgery, Cologne-Merheim Hospital, Kliniken der Stadt Köln gGmbH, Witten-Herdecke University Hospital, Cologne, Germany

Correspondence to

Dr Maximilian Ackermann, Institute of Functional and Clinical Anatomy, University Medical Center of the Johannes Gutenberg University Mainz, Johann-Joachim-Becher-Weg 13, Mainz 55128, Germany: maximilian.ackermann@unimainz.de

Received 10 August 2016 Revised 15 November 2016 Accepted 28 November 2016 Published Online First 2 January 2017

CrossMark

To cite: Ackermann $\mathrm{M}_{\text {, }}$ Mentzer SJ, Roth W, et al. Thorax 2017;72:861-863.
A 24-year-old Caucasian man was admitted with a known hereditary haemorrhagic telangiectasia (HHT) and heterozygous mutation of factor $\mathrm{V}$ Leiden following episodes of cerebral infarctions in occipital lobes, cerebellum and brainstem. In his

case history, the patient underwent several interventional embolisation of arteriovenous (AV) malformations in the middle and lower lobes (figure 1). However, those were not completely successful as the malformations were diffuse. We performed

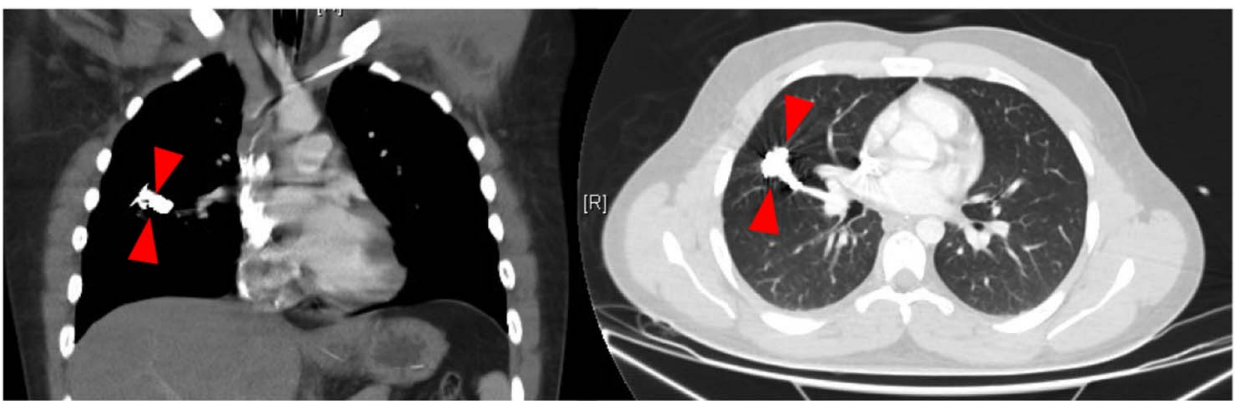

Figure 1 CT scans depict the pulmonary arteriovenous malformations after re-embolisation in the middle lobe.
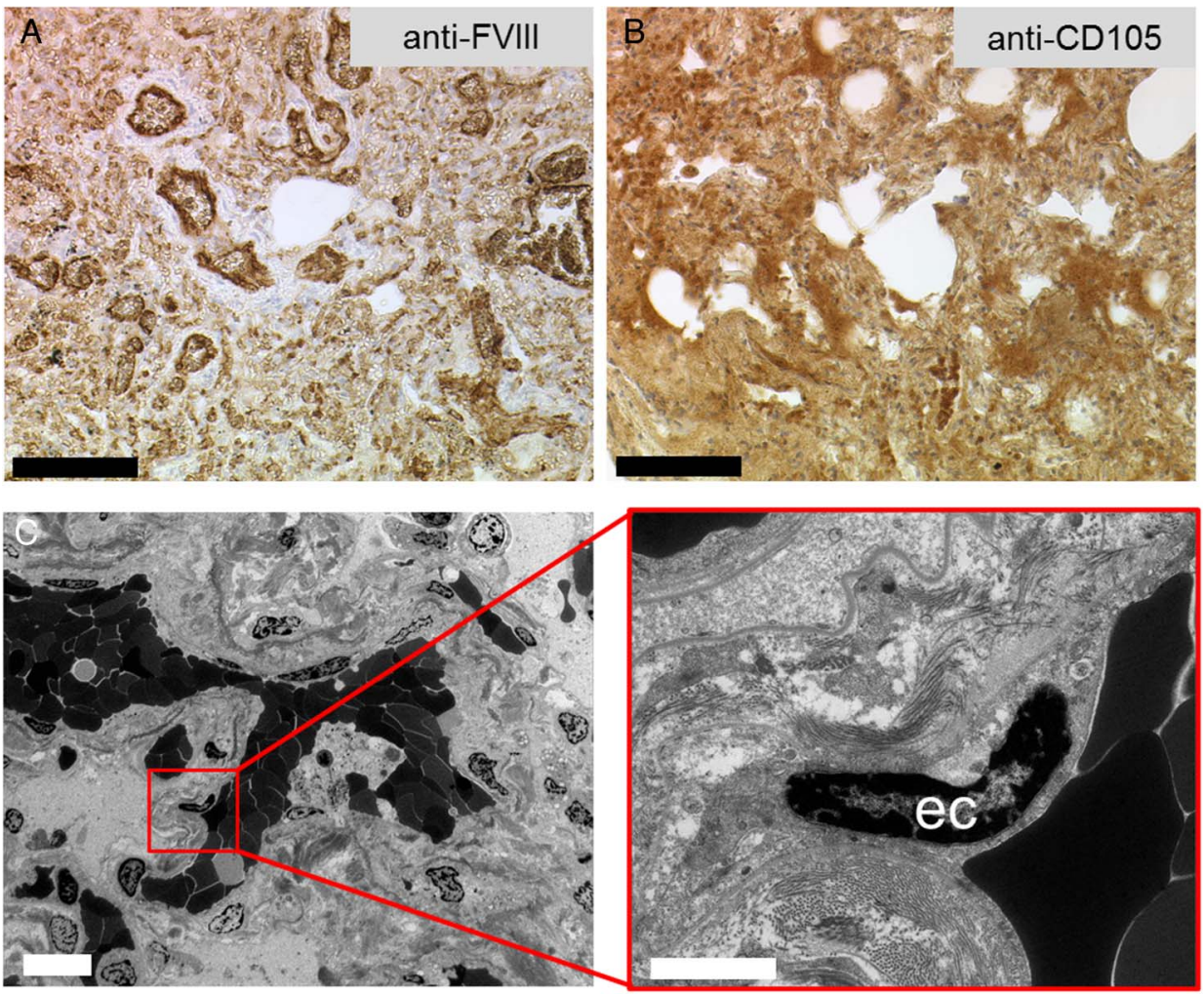

Figure 2 (A and B) Immunohistochemical staining against FVIII antigen (endothelial cells (ec)) and CD105 (endoglin) exposed a high expression of endoglin in the hereditary haemorrhagic telangiectasia (HHT) lung, bar=100 $\mu \mathrm{m}$ (C). Transmission electron microscopy displayed a high vascularity and the appearance of sprouting angiogenesis, bars $=10 \mu \mathrm{m}, 2 \mu \mathrm{m}$. 

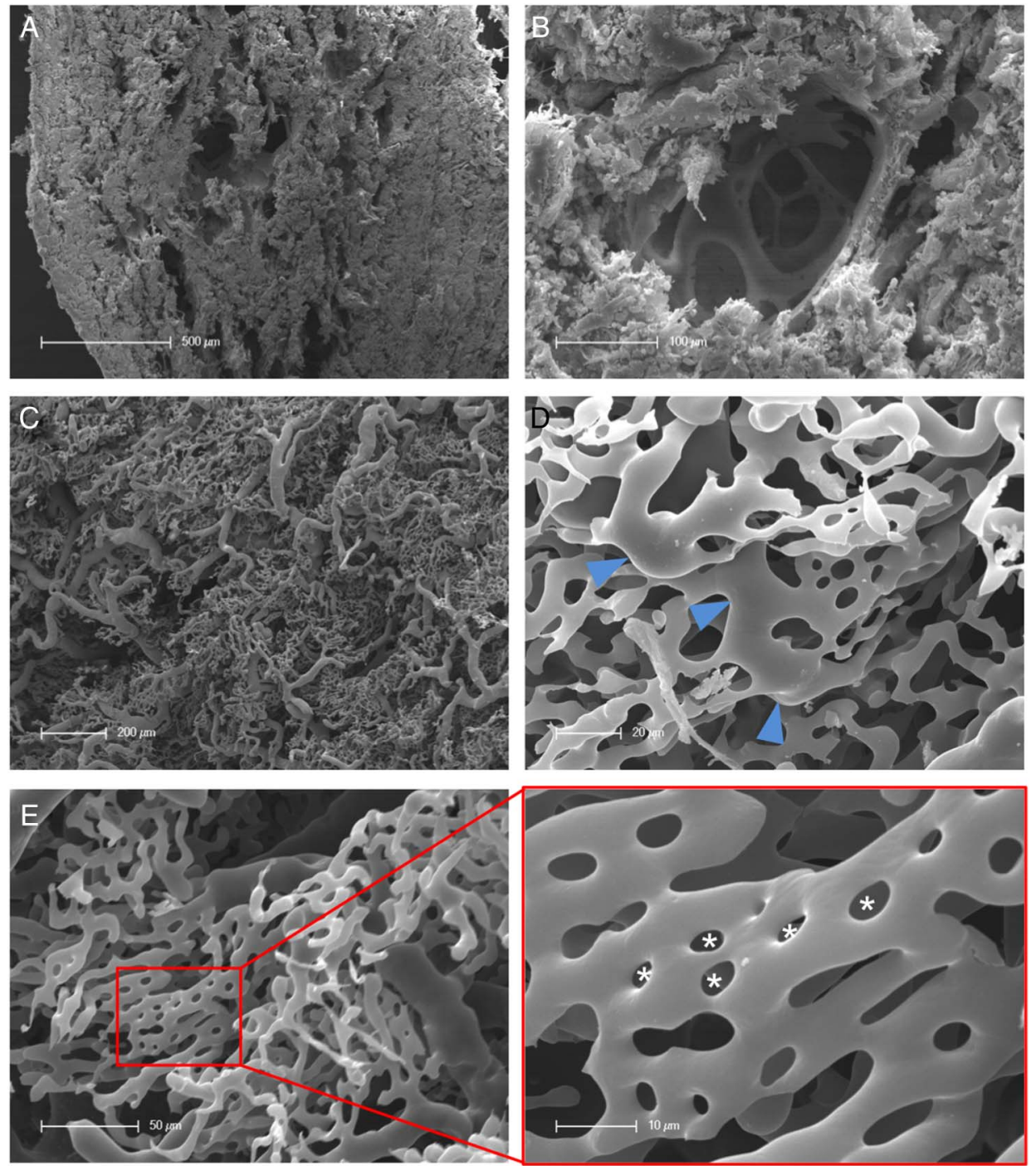

Figure 3 Structural morphology of hereditary haemorrhagic telangiectasia (HHT) lung tissue and vascularity. (A) Cross section of HHT middle lobe exhibiting pronounced consolidated areas with haemorrhages. (B) This architectural distortion leads to an obliteration of an alveolar duct. (C) Microvascular corrosion casting exposed areas with densely packed aberrant bulks of pulmonary arteriovenous malformations (PAVMs). (D) The branches of varying calibre and dilated vessels (arrowheads) reflecting the chaotic perfusion caused by the PAVM shunting. (E) The expansion of pulmonary capillary plexus is mainly driven by intussusceptive angiogenesis as evidenced by the appearance of numerous tiny holes (exemplary marked with*).

video-assisted thoracoscopic surgery with a resection of the middle lobe and a wedge resection of segment 10.

HHT (Osler-Weber-Rendu disease) is an autosomal-dominant disease determined by multiple dilated vessels including mucocutaneous telangiectasias and vascular malformations of visceral organs resulting in recurrent epistaxis, gastrointestinal bleedings, paradoxical emboli, cerebral infarctions and abscesses. ${ }^{1}$ In about $55 \%$ of patients with HHT type 1 (HHT-1) and about $10 \%$ of patients with HHT type 2 (HHT-2), large pulmonary arteriovenous malformations (PAVMs) are found which results in a direct shunting of arterial and venous blood flow. ${ }^{2}$ These PAVMs cause a decreased resistance and enhanced likelihood of pulmonary haemorrhage. The underlying pathogenetic mechanism of HHT is associated with loss-of-function mutations of endoglin (HHT-1) and ALK-1 (HHT-2). However, the precise underlying mechanisms of HHT on the pulmonary vascular architecture have not been reported yet in the literature.

To define the pulmonary vascular architecture in this patient, the harvested lung tissue of middle lobe was analysed by microvascular corrosion casting, ${ }^{3}$ transmission electron microscopy and immunohistology. Histologically, we identified dense nodular capillary proliferations (figure $2 \mathrm{~A}$ ). In particular, the immunohistological staining against endoglin (CD105), the HHT-relevant transforming growth factor beta-binding protein, demonstrated profuse expression in these capillary proliferations (figure 2B), whereby HHT-1 is usually associated with a haploinsufficiency of endoglin. Contrary to the high endoglin expression within these proangiogenic PAVMs, we realised a weak staining in the surrounding tissue. Notably, histopathological observations on human PAVMs could show that endoglin was still expressed in the AV malformation suggesting that the lesion is not caused by a loss of heterozygosity. ${ }^{3}$ It may be assumed that the PAVMs are active hot spots of pulmonary angiogenesis and remodelling, which reveal a focal upregulation of endoglin activity in these vascular alterations. The PAVMs with several AV shunting resulting in hypoxia and upregulation of angiogenesis might recruit circulating endothelial precursor cells. The ultrastructural analysis of these $\mathrm{AV}$ malformations by transmission electron microscopy 
highlighted dilated, thin-walled capillaries causing multiple diffuse alveolar haemorrhages which compress the surrounding lung tissue (figure 3A, B). When looking at the alterations of pulmonary vascular structure, the vascular architecture in the middle lobe is characterised by numerous PAVMs forming a tangled vascular network structure (figure 3C). However, the expanding capillary plexus indicate a distortion of vascular hierarchy with dilated, elongated capillaries and sudden changes of calibre abruptly draining into thinned vascular tufts (figure 3D). The analysis of microvascular architecture revealed the morphological evidence of both sprouting (figure 2C) and intussusceptive angiogenesis (figure $3 \mathrm{E}$ ). Intussusceptive (non-sprouting) angiogenesis is a well-characterised rapid morphogenetic process observed during the capillary expansion in cancer, inflammation and regeneration, which is distinct from sprouting angiogenesis because it has no necessary requirement for cell proliferation. ${ }^{4}$ In this respect, mechanical stress, the excessive recruitment of pericytes and smooth muscle cells related to the formation of PAVMs and the changes in blood flow are thought to play pivotal roles in the initiation of the intussusceptive microvascular growth. These visual insights into the pulmonary microvascular alterations in
HHT help explain the clinical manifestations of the disease as well as illuminate the challenges of potential multitargeted antiangiogenic therapy.

Contributors ES managed the patient. MA prepared the manuscript. MA and WR prepared the images. MA, UG and SJM revised the manuscript.

Competing interests None declared.

Patient consent Obtained.

Provenance and peer review Not commissioned; externally peer reviewed.

\section{REFERENCES}

1 Shovlin CL, Letarte M. Hereditary haemorrhagic telangiectasia and pulmonary arteriovenous malformations: issue in clinical management and review of pathogenic mechanisms. Thorax 1999;54:714-29.

2 Cottin V, Dupuis-Girod S, Lesca G, et al. Pulmonary vascular manifestations of hereditary hemorrhagic telangiectasia (Rendu-Osler disease). Respiration 2007;74:361-78.

3 Bourdeau A, Cymerman U, Paquet ME, et al. Endoglin expression is reduced in normal vessels but still detectable in arteriovenous malformations of patients with hereditary hemorrhagic telangiectasia type 1. Am J Pathol 2000;156:911-23.

4 Ackermann $M$, Konerding MA. Vascular casting for the study of vascular morphogenesis. Methods Mol Biol 2015;1214:49-66. 